\title{
Children and pregnant women are more likely to develop severe Mycoplasma pneumoniae Pneumonia冈Mycoplasma pneumoniae infection in Suzhou: 2014 to 2018
}

yantian Lv ( $\sim$ lvyantian@126.com )

First Affiliated Hospital of Soochow University

\section{Xiaojing Sun}

First Affiliated Hospital of Soochow University

\section{Ying Chen}

Nanjing Medical University Affiliated Suzhou Hospital: Suzhou Municipal Hospital

\section{Ting Ruan}

Nanjing Medical University Affiliated Suzhou Hospital: Suzhou Municipal Hospital

\section{Guopeng Xu}

Nanjing Medical University Affiliated Suzhou Hospital: Suzhou Municipal Hospital

\section{Xu Xiao}

Nanjing Medical University Affiliated Suzhou Hospital: Suzhou Municipal Hospital

Jianan Huang

First Affiliated Hospital of Soochow University

\section{Research Article}

Keywords: Mycoplasma pneumoniae, Respiratory tract infection, Children, Adult, Pregnant women, Severe Mycoplasma pneumoniae pneumonia

Posted Date: February 16th, 2021

DOI: https://doi.org/10.21203/rs.3.rs-221498/v1

License: (c) (1) This work is licensed under a Creative Commons Attribution 4.0 International License. Read Full License 


\section{Abstract \\ Purpose}

To study the epidemic characteristics of Mycoplasma pneumonia(MP) in the whole population and the clinical characteristics of severe Mycoplasma pneumoniae pneumonia(SMMP).

\section{Methods}

We retrospectively analyzed 56474 patients with respiratory tract infections (RTIS) of the affiliated hospital of Nanjing medical university from 2014 to 2018. Serum particle agglutination (PA) was used to detect MP specific IgM antibody to confirm MP infection.

\section{Results}

A total of 56474 patients with RTIs were enrolled, and 15857 (28.13\%) of patients with MP-IgM positive were detected. From 2014 to 2018, the annual proportion of MP RITs was $23.60 \%, 28.18 \%, 38.08 \%$, $27.05 \%$ and $23.44 \%$. In terms of seasonal distribution, April-June and September-November were the peak seasons each year. The proportion of MP RITs of children was significantly higher than that of adults in almost every year. The proportion of MP RITs of female was significantly higher in children and adults. The peak age of MP infection was between the age of 4 and 14 years old. There were 216 cases of SMPP patients, including 198 children and 18 adults (8 cases of pregnant women). Children and pregnant women were more likely to develop severe Mycoplasma pneumonia. SMMP patients had more extrapulmonary symptoms, multilobar infiltrates and pleural effusion, and increased CRP, LDH and $\operatorname{WBC}(p<0.05)$.

\section{Conclusions}

This study shows that MP infection has a seasonal, gender, and age distribution trend, and SMMP is more likely to occur in children and pregnant women. Understanding these characteristics will contribute to timely diagnose and treat MP infection.

\section{Background}

Mycoplasma pneumoniae (MP) is a common pathogen of respiratory tract infections worldwide, usually sporadic, and there is a regional epidemic every 3 to 5 years, and each epidemic lasts for 1 to 2 years ${ }^{[1,2]}$. The clinical manifestations of patients can be mild self-limited or transform into imaging-confirmed pneumonia requiring hospitalization, accounting for approximately $10-30 \%$ of community-acquired pneumonia (CAP) cases $^{[3-5]}$, in severe cases, it can cause multiple organ damage ${ }^{[6]}$. It is of great 
significance to monitor the epidemic trend of MP infection and analyze the epidemic characteristics. As

so far, the domestic researches on the epidemiological characteristics mainly focus on the children ${ }^{[7-9]}$, data on epidemiology of whole population of MP infection in China are little known, especially in certain special populations, such as pregnant women. Therefore, in order to better understand the epidemiological characteristics of MP in Suzhou, we collected data on the whole population of MPinfected patients in outpatients and inpatients over the past 5 years to analyze the distribution of gender, age, season and other factors.

\section{Material And Methods}

\section{Study subjects}

From January 2014 to December 2018, a total of 56474 outpatients and inpatients with respiratory tract infections, aged 0-94 years (median age 12 years), including 28791 males and 27683 females(37828 children and 18936 adults), were enrolled in the outpatient and inpatient clinics of the affiliated suzhou hospital of Nanjing medical university. Demographic features of the patients' clinical information and laboratory data were retrospectively collected from the records of all patients. The MP Respiratory tract infections (RTIs) included upper tract infection, bronchitis, bronchopneumonia, and pneumonia. The study protocol was approved by the Medical Research Ethics Committee of affiliated hospital of Nanjing medical university, strictly abiding by the declaration of the Declaration of Helsinki. The requirement for informed consent was waived by the committee as our study was an anonymous retrospective review of patient records.

\section{Detection of Mycoplasma pneumoniae and protocols}

For outpatients: patients with dry cough(with or without fever, peripheral white blood cell count $<10000$ / $\mu \mathrm{l})$ more than 1 week (or family members with cough), will be advised for MP-specific IgM test. For inpatients: all admitted patients underwent MP-specific IgM test routinely. MP infection was confirmed by detection of MP-specific IgM $\triangle S E R O D I A \circledast-M Y C O \|$ II,Fujirebio $\otimes$, which is defined as a single serum MPspecific IgM titer greater than 1:320, or a second seroconversion (a 4-fold increase in antibody titer) ${ }^{[10-12]}$.

\section{Respiratory tract infections (RTIs) diagnosis}

RTIs including upper tract infection, bronchitis, bronchopneumonia, and pneumonia were diagnosed based on clinical symptoms (fever, sore throat, cough, sputum and wheezing, etc.) and imaging infiltrates. Severe Mycoplasma pneumoniae pneumonia(SMMP) defined as pneumonia with one of the followings: persistent fever $>38.5 \mathrm{C}$ for more than 10 days, radiological deterioration or consolidation present in $>2 / 3$ of the lung lobes, and intra- and extrapulmonary complications, pulse oxygen less than $92 \%$, shock, referring to the criteria for severe pneumonia in children and adults with community-acquired pneumonia ${ }^{[13,14]}$.

\section{Statistical analysis}


Data expressed in terms of the mean, count, and percentage. Data analysis was performed using the SPSS 21.0 statistical software package (IBM Corp., Armonk, NY, USA). Continuous data are expressed as mean \pm standard deviation. Continuous variables are analyzed by Student's $t$ test, and categorical variables are analyzed by $\chi 2$ test or Fisher's exact test. $P$ value $<0.05$ was considered significant.

\section{Results}

\section{Total epidemic characteristics in 2014-2018}

A total of 56474 patients with RITS were suspected of MP infection, of which 15857 cases were positive for MP-IgM, and the total MP infection proportion was $28.13 \%$. The main clinical symptoms were cough, headache, sore throat, nasal symptoms, fever, malaise, and few of extra-pulmonary symptoms such as skin rash, meningitis, mycocarditis, hemolytic anemia, myalgias digestive symptoms etc. The MP RITs proportion from $2014-2018$ were $23.60 \%, 28.18 \%, 38.12 \%, 27.05 \%$ and $23.44 \%$ respectively. The MP infection proportion was highest in 2016 and lowest in 2018 (as shown in Figure 1 and Table 1).

\section{Children and adults of Respiratory tract infections with Mycoplasma pneumoniae}

As shown in Figure 1 and Table 1, except for individual months, the MP RITS proportion in children was significantly higher (approximately 2 folds) than that in adults in almost every month. The MP RITS proportion from $2014-2018$ of children and adults were $26.91 \%$ vs $16.02 \%, 36.56 \%$ vs $11.78 \%, 46.28 \%$ vs $18.19 \%, 37.44 \%$ vs $13.65 \%, 27.91 \%$ vs $14.95 \%$ respectively. And the epidemic trends of the two groups were consistent.

\section{Gender distributions of Respiratory tract infections with Mycoplasma pneumoniae}

In order to explore the gender distribution of MP infection, we distinguish it between children and adults. As shown in Table1, the MP RITs proportion of girls and boys were $40.28 \%$ vs $31.87 \% \%$ in children, and $21.67 \%$ vs $9.50 \%$ of females and males in adults. The MP RITs proportion in female were consistently elevated in both children and adults $(P<0.0001)$

\section{Season distributions of Respiratory tract infections with Mycoplasma pneumoniae}

In terms of seasonal distribution, MP was prevalent from January to December, and there was no obvious trough, but April to June and September to November were relatively peak months, especially in the epidemic year of 2016 .The MP RITs proportion was $44.4 \%$ to $49.7 \%$ from April to June, and $37.6 \%$ to 48.7\% from September to November in 2016 (as shown in Figure 2).

\section{Age distributions of Respiratory tract infections with Mycoplasma pneumoniae}

Our study included the entire population of 0-94 years old. Because MP is mainly prevalent in children, the age group interval of children under 14 years old was 1 year, while people aged 14 or above were based on different age intervals. According to the age distribution, children and adolescents were the main 
population of MP infection, of which aged 4-14 years old were the high-risk group. The MP RITs proportions were from $37.34 \%$ to $51.42 \%$. In adults, the MP RITs proportion decreased gradually after the 20 years old, and the MP RITs proportion of elderly patients was very low, less than $5 \%$ (as shown in Figure 3).

\section{MPP and SMPP in children and adults, pregnant and non-pregnant women}

As shown in Table 2, from 2014 to 2018, there were 1465 cases of MPP including 1241 case of children and 223 cases of adults, 216 cases of SMPP, including 198 cases of children and 18 cases of adults. A total of 504 pregnant women and 528 non-pregnant women of reproductive age were enrolled during the period. The MP RITs proportion in women and non-pregnant women were $120 / 504(23.81 \%)$ vs $142 / 528(26.90 \%)$. There were 35 cases of MPP (8 cases of SMMP) in pregnant women, 30 cases of MPP (including 2 cases of SMMP) in non-pregnant women. SMPP accounted for a higher proportion in children and pregnant women than that of adults and non-pregnant women $(p=0.0403)$.

\section{Clinical characteristics of MPP and SMPP in adults and children}

We further analyzed MMP and SMMP related clinical presentation, laboratory detection and image manifestations. As shown in table 3, SMMP patients have more extra-pulmonary symptoms than MMP patients, which may be related to the secondary immune response of Mycoplasma pneumoniae. In addition, patients with SMMP had more multilobar infiltration and pleural effusion, and increased CRP, LDH and Leukocyte Count. In addition, in our study, pregnant women accounted for nearly half of the number of patients with SMMP, and their symptoms tend to develop mutilobar infiltration and pleural effusion and respiratory failure more rapidly (as shown in Figure 4).

\section{Discussion}

Mycoplasma pneumonia (MP) is a prokaryotic microorganism with a size between bacteria and viruses, which can cause epidemics worldwide. In epidemic years, MP can account for $30-50 \%$ of RTIs ${ }^{[15]}$. Since 2010, MP outbreaks have been reported in several countries, including Europe, the United States, China and Japan ${ }^{[2,16,17]}$. From 2014 to 2018 , we observed that $28.07 \%$ (15857 /56474) of patients with RTIs were positive for MP, which was higher than the global incidence as $12 \%$ (range $11-15 \%$ ) from the Atypical Pathogens Reference Laboratory Database ${ }^{[17]}$. However, the data are basically consistent with the studies in China and Asia ${ }^{[2,7,8,19,20]}$.Our research shows that the MP RITs proprotion in the population increased significantly in 2016 , and even exceeded $50 \%$ during the epidemic season. In nonepidemic years, the average MP infection rate is also around $25 \%$, considered to be related to the widespread presence of Macrolide-Resistant MP in Asia.

MP infection is sporadic throughout the year, but studies have shown that the peak epidemic seasons are distinctive in different areas ${ }^{[21]}$. Studies from South Korea show that MP infections are more common in autumn and winter ${ }^{[22]}$. European data indicate that June and July are the peak epidemic seasons of MP 
$[23,24]$. However, researches from Seattle demonstrate no seasonal difference in MP infections ${ }^{[25]}$.In China, Gao and Qu reported that the peak epidemic seasons of MP are dominated in autumn and winter in the north, summer and autumn in the south ${ }^{[26,27]}$. Our results show that April to June and July to September are the peak months of the MP epidemic, which is consistent with the data of Qian and Zhang ${ }^{[20,28]}$. According to the epidemic situation of various countries, summer and autumn are the main peak seasons of MP epidemic.

In our study, females are more likely to be infected with MP than males in both children and adults each year, which is consistent with previous research ${ }^{[8,9,25-28]}$. A reasonable explanation may be related to the different levels of activities and immunity between female and male. For children, boys have more outdoor sports than girls, especially in school-age Children, and girls are more likely to have indoor activities during recess, while indoor activities are relatively closed. Long time indoor activities are more conducive to MP transmission. For adults, a lower level of immunity in female than male may be a factor in susceptibility to MP.

As shown in Fig. 1, The MP RITs proportion in children were significantly higher than that in adults almost every month. The peak epidemic month of MP infection in children and adults were from Apr to Jun and Sep to Nov. Similarly, Qu et al ${ }^{[26]}$ found that the positive rate of MP in children was significantly higher than that of adults $(19.7 \%$ and $8.9 \%, P<0.001)$, the highest rate was found in the school children group aged 7-17 years. Kogoj et al ${ }^{[29]}$ reported that the proportion of MP infection in children and adults was $19 \%$ and $7 \%$, respectively, and children with 6-16 years old were at serious risk of MP infection. In terms of age distribution, we found that children aged 4-14 years old are the main risk population, which is identical to the previous studies ${ }^{[26,29]}$. The pathogenic mechanism of MP infection is not only the direct damage to human body, but also the damage mediated by immune response ${ }^{[30]}$. The immune system of infants and young children is immature, and the immune response level is low. Therefore, infants younger than 3 years old show mild infection or subclinical infection ${ }^{[15]}$. The high MP RITs proportion among children over 4 years old may be closely related to their long-term stay in semi-closed venues such as kindergartens and schools.

In our study, we also included some pregnant women. Pregnant women have attracted much attention because of their special physiological characteristics. A total of 504 pregnant women enrolled in this study during the past 5 years. Compared with 528 contemporaneous non-pregnant women, the MP RITs proportion in pregnant women had no significant difference. But SMPP accounted for a higher proportion in pregnant women than that non-pregnant women( $29.62 \%$ vs $7.14 \%, p=0.0403)$.

MPP is mostly mild and has a good prognosis, but the SMMP has severe manifestations, long course of disease, multiple complications and sequelae such as atelectasis, bronchiectasis, bronchiolitis obliterans and bronchiolitis obliterans. Our study found that SMMP patients had more extrapulmonary manifestations such as skin rash, meningitis, mycocarditis, hemolytic anemia, myalgias digestive symptoms etc. The main pathogenesis are direct damage and indirect immune response ${ }^{[30,31]}$. In 
addition to endothelial cells, MP can adhere to other cells, such as red blood cells and macrophages, these cells enter multiple organs and cause tissue damage. In addition, MP activates some toll-like receptors, in particular TLR2, which can provide also damage-associated molecular patterns, promoting the initiation of inflammatory responses ${ }^{[31]}$. Moreover, our research showed that the proportion of SMMP in children is much higher than that of adults. Miyashita ${ }^{[32]}$ counted 227 adult patients with MPP, 13 of which were severe SMMP, accounting for $5.7 \%(13 / 227)$. Gao ${ }^{[28]}$ reported that SMPP made up about $13.0 \%$ of all cases of MPP. It has urgent clinical value to early identify SMMP in younger patients with MPP. Studies showed that more than 10 days of fever, younger children, lung consolidation, massive pleural effusion, necrotizing pneumonia, CRP > $60 \mathrm{mg} / \mathrm{L}, \mathrm{LDH}>410 \mathrm{IU} / \mathrm{L}$, and extra-pulmonary complications can be used as indicators to predict SMPP ${ }^{[33-35]}$.

In children, atelectasis, pleural effusion, necrotizing pneumonia or lung abscess are common in severe SMMP patients ${ }^{[36]}$. Due to the special status of pregnant women, general imaging examination is not as a routine examination. In clinical practice, according to the recommendations of Obstetrics and gynecology in the United States ${ }^{[37]}$, we will also recommend imaging examination for pregnant women with poor response to treatment. Our retrospective analysis showed that pregnant women with SMMP also had the characteristics of multilobar infiltration and pleural effusion, and the progress was often rapid.

Restricted by the retrospective design of the study, many medical data such as drug use history, efficacy, social and economic factors cannot be analyzed. In the diagnosis of MP, limited by our hospital's own conditions, we failed to carry out PCR testing, and there may be some bias in the interpretation of the results. Compared with other populations, the number of pregnant women is still relatively small in our study, and more cases need to be enrolled, and more information about the fetus needs collecting to analyze the relationship between Mycoplasma infection and pregnancy outcome. However, because this study contains sufficient samples and has been continuously monitored for 5 years, which will make our conclusion reliable.

\section{Conclusions}

In summary, our research shows that MP infection is very common in Suzhou. MP infection peaks will appear from April to June and from September to November every year. Women and children are susceptible to MP. Children and adolescents aged 4-14 years old are the main populaiton with MP. Pregnant women and children are more likely to develop severe Mycoplasma pneumonia. Some clinical indicators are needed to early identify of SMMP.

\section{Declarations}

\section{Authors' contributions}


Yantian Iv and Jianan Huang were responsible for the organization and coordination of the clinical data. Yantian Iv and Xiaojing Sun were the chief investigators and responsible for the data analysis. Yantian Lv, Xiaojing Sun, Ying Chen, Ting Ruan, Guopeng Xu and Xiao Xu collected clinical data. All authors contributed to the writing of the final manuscript

\section{Funding}

This study was supported by grant kjxw2018027 (Suzhou science and Technology Bureau).

\section{Ethics and informed consent}

The requirement for informed consent was waived by the committee of Affiliated Suzhou Hospital of Nanjing Medical University as our study was an anonymous retrospective review of patient records and there was no potentially identifiable human images or data presented in this study. The study was approved by the committee of Affiliated Suzhou Hospital of Nanjing Medical University.

\section{Conflict of interest}

All the authors have no conflicts of interest to declare.

\section{Availability of data and material}

The datasets used and/or analyzed during the current study are available from the corresponding author on reasonable request.

Code availability: Not applicable.

\section{References}

[1] Chaudhry R, Ghosh A, Chandolia A. Pathogenesis of MP: An update. Indian J Med Microbiol. 2016. 34(1): 7-16.

[2] Yamazaki T, Kenri T. Epidemiology of MP Infections in Japan and Therapeutic Strategies for Macrolide-Resistant M. pneumoniae. Front Microbiol. 2016. 7: 693-707.

[3] Cao B, Zhao CJ, Yin YD, Zhao F, Song SF. High prevalence of macrolide resistance in MP isolates from adult and adolescent patients with respiratory tract infection in China. Clin Infect Dis. 2010. 51(2): 189194.

[4] Eibach D, Casalegno JS, Escuret V, G Billaud冈F Morfin. Increased detection of MP infection in children, Lyon, France, 2010 to 2011. Euro Surveill. 2012. 17(8):2-4.

[5] Brown RJ, Macfarlane-Smith L, Phillips S, Chalker VJ. Detection of macrolide resistant MP in England, September 2014 to September 2015. Euro Surveill. 2015. 20(48): 30078. 
[6] Bajantri B, Venkatram S, Diaz-Fuentes G. MP: A Potentially Severe Infection. J Clin Med Res. 2018. 10(7): 535-544.

[7] Yan C, Sun H, Zhao H. Latest Surveillance Data on MP Infections in Children, Suggesting a New Epidemic Occurring in Beijing. J Clin Microbiol. 2016. 54(5): 1400-1401.

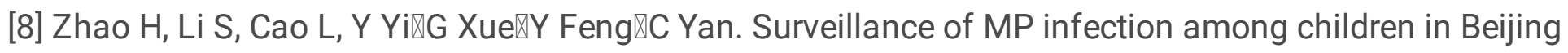
from 2007 to 2012. Chin Med J (Engl). 2014. 127(7): 1244-1248.

[9] Gao J, Xu L, Xu B, Xie Z, Shen K. Human adenovirus Coinfection aggravates the severity of MP pneumonia in children. BMC Infect Dis. 2020. 20(1): 420-424.

[10] Respiratory Branch of Chinese Pediatric Society of Editorial Board of Chinese Journal of Applied Clinical Pediatrics (2015) Expert consensus on diagnosis and treatment of MP pneumonia in children. Chinese Journal of Applied Clinical Pediatrics 30,1304-1308.

[11] Meyer Sauteur P M , Unger W W J , Van Rossum A M C , C Berger. The Art and Science of Diagnosing Mycoplasma pneumoniae Infection[J]. Pediatr Infect Dis J. 2018 Nov;37(11):1192-1195.

[12] Clinical Laboratory Science Group of Pediatrics Branch of Chinese Medical Association. Consensus of Chinese experts on laboratory diagnosis of MP respiratory tract infection in children $₫$ Chin $\mathrm{J}$ Lab Med. 2019,42 (7):507-513区

[13] Salih W, Schembri S, Chalmers JD. Simplification of the IDSA/ATS criteria for severe CAP using metaanalysis and observational data. Eur Respir J. 2014. 43(3): 842-851.

[14] Subspecialty Group of Respiratory Diseases, The Society of Pediatrics, Association CM, Editorial Board, Chinese Journal of Pediatrics. [Guidelines for management of community acquired pneumonia in children (the revised edition of 2013) (I)]. Zhonghua Er Ke Za Zhi. 2013. 51(10): 745-752.

[15] Waites KB, Xiao L, Liu Y, Balish MF, Atkinson TP. MP from the Respiratory Tract and Beyond. Clin Microbiol Rev. 2017. 30(3): 747-809.

[16] Brown RJ, Nguipdop-Djomo P, Zhao H, Stanford E, Spiller OB, Chalker VJ. MP Epidemiology in England and Wales: A National Perspective. Front Microbiol. 2016. 7: 157.

[17] Xue G, Li M, Wang N, J Zhao \W BeiðZ Ren囚et al. Comparison of the molecular characteristics of MP from children across different regions of China. PLoS One. 2018. 13(8): e0198557:1-10.

[18] Arnold FW, Summersgill JT, Lajoie AS, P Peyrani囚Julio A Ramirez. A worldwide perspective of atypical pathogens in community-acquired pneumonia. Am J Respir Crit Care Med. 2007. 175(10): 1086-1093.

[19] Lee E, Cho HJ, Hong SJ, Lee J, Sung H, Yu J. Prevalence and clinical manifestations of macrolide resistant MP pneumonia in Korean children. Korean J Pediatr. 2017. 60(5): 151-157. 
[20] Zhang Xx. Epidemiological analysis of MP infection in children with respiratory tract diseases in Suzhou area from 2005 to 2014. China Medical Abstracts (Internal Medicine). 2016. v.33(1): 15-16.

[21]Onozuka D, Hashizume M, Hagihara A. IMPact of weather factors on MP pneumonia. Thorax. 2009. 64(6): 507-511.

[22] Eun BW, Kim NH, Choi EH, Lee HJ. MP in Korean children: the epidemiology of pneumonia over an 18year period. J Infect. 2008. 56(5): 326-331.

[23] Defilippi A, Silvestri M, Tacchella A, R Giacchino $\ G A$ Rossi. Epidemiology and clinical features of MP infection in children. Respir Med. 2008. 102(12): 1762-1768.

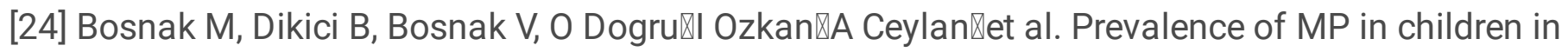
Diyarbakir, the south-east of Turkey. Pediatr Int. 2002. 44(5): 510-512.

[25] Foy HM, Kenny GE, Cooney MK, Allan ID. Long-term epidemiology of infections with MP. J Infect Dis. 1979. 139(6): 681-687.

[26] Qu J, Yang C, Bao F, Chen S, Gu L, Cao B. Epidemiological characterization of respiratory tract infections caused by MP during epidemic and post-epidemic periods in North China, from 2011 to 2016. BMC Infect Dis. 2018. 18(1): 335-338.

[27] Gao LW, Yin J, Hu YH, XY LiuヌKL Shen. The epidemiology of pediatric MP pneumonia in North China: 2006 to 2016. Epidemiol Infect. 2019. 147: e192:1-5.

[28] Qian Q, Ji W. Epidemiological features of children hospitalized with mycoplasma pneumoniae infection from the year of 2006 to 2014.Chongqing Med 2016. 45(29): 4113-4116.

[29] Kogoj R, Mrvic T, Praprotnik M, Kese D. Prevalence, genotyping and macrolide resistance of MP among isolates of patients with respiratory tract infections, Central Slovenia, 2006 to 2014. Euro Surveill. 2015. 20(37).

[30] He J, Liu M, Ye Z, Tan TP,Hui X,Xiao X. Insights into the pathogenesis of MP (Review). Mol Med Rep. 2016.14(5): 4030-4036.

[31]Dimitri, Poddighe. Extra-pulmonary diseases related to Mycoplasma pneumoniae in children: recent insights into the pathogenesis[J]. Curr Opin Rheumatol.2018 Jul;30(4):380-387.

[32] Miyashita N, Obase $\mathrm{Y}$, Ouchi K, K Kawasaki $\ Y$ Kawai $\ Y$ Kobashi囚et al. Clinical features of severe MP pneumonia in adults admitted to an intensive care unit. J Med Microbiol. 2007. 56(Pt 12): 1625-1629.

[33] Wang CY, Wang SB, Liu GH, Song CM, Chen Q. Analysis of correlations between clinical characteristics and pediatric critical illness score in children with severe Mycoplasma pneumoniae pneumonia. Chin J Evid Based Pediatr.2016.11(4): 280-284. 
[34] Jing XY, Lu M. Risk factors and clinical significance of severe mycoplasma pneumoniae pneumonia. Int J Pediatr. 2016. 43(7): 519-522.

[35] Yan C, Xue G, Zhao H, Y Feng $₫$ S Li囚J Cui囚et al. Molecular and clinical characteristics of severe MP pneumonia in children. Pediatr Pulmonol. 2019. 54(7): 1012-1021.

[36] Robnik B, Kese D, Rojko T, A Horvat-Ledinek囚Pranikar, Ale®B Beovic. Unilateral brachial plexopathy, a rare complication of Mycoplasma pneumoniae infection[J]. J Infect Chemother. 2018 Apr; 24(4):309-311.

[37] American College of Obstetricians and Gynecologists' Committee on Obstetric Practice. Obstet Gynecol. 2016;127(2):e75-80.

\section{Tables}

Table 1 Total epidemic characteristics of MP infection in adults and children from 2014 to 2018.

\begin{tabular}{|c|c|c|c|c|}
\hline & Total(n,\%) & children(n,\%) & adult(n,\%) & $P^{*}$ \\
\hline \multicolumn{5}{|l|}{ Year } \\
\hline 2014 & $1885 / 7988(23.60 \%)$ & 1495/5554ه26.91\%区 & $390 / 2434 \rrbracket 16.02 \% \rrbracket$ & $<0.0001$ \\
\hline 2015 & $2985 / 10593(28.18 \%)$ & $2563 / 7011 \rrbracket 36.56 \% \rrbracket$ & $422 / 3582 \otimes 11.78 \% \bigotimes$ & $<0.0001$ \\
\hline 2016 & 4188/10998(38.08\%) & $3574 / 7723 \llbracket 46.28 \% \rrbracket$ & 614/3375囚18.19\%》 & $<0.0001$ \\
\hline 2017 & $3707 / 13704(27.05 \%)$ & $3025 / 8709 \llbracket 37.44 \% \rrbracket$ & 682/4995囚13.65\%区 & $<0.0001$ \\
\hline 2018 & $3092 / 13191(23.44 \%)$ & $2412 / 8641 \llbracket 27.91 \% \bigotimes$ & $680 / 4550 \otimes 14.95 \% \rrbracket$ & $<0.0001$ \\
\hline \multicolumn{5}{|l|}{ Gender } \\
\hline male & $6758 / 28791(23.47 \%)$ & $5732 / 17986 \rrbracket 31.87 \% \rrbracket$ & $1026 / 10805 \rrbracket 9.50 \% \rrbracket$ & $<0.0001$ \\
\hline female & 9639/27683(34.82\%) & $7877 / 19552 \rrbracket 40.28 \% \bigotimes$ & 1762/8131区21.67\%】 & $<0.0001$ \\
\hline$P^{\#}$ & $<0.0001$ & $<0.0001$ & $<0.0001$ & \\
\hline
\end{tabular}

MP: Mycoplasma pneumoniae * compaired to children and adults, \# compaired to male and female Table 2 Epidemic characteristics of MPP and SMPP in children and Adults 


\begin{tabular}{|llll|}
\hline & MP RITS (n,\%) & MPP $(\mathrm{n}, \%)$ & $\operatorname{SMPP}(\mathrm{n}, \%)$ \\
\hline Children & $13069 / 37488(34.86 \%)$ & $1241 / 13069(9.50 \%)$ & $198 / 1241(15.95 \%)$ \\
\hline Adult & $2788 / 18996(14.68 \%)$ & $223 / 2788(8.00 \%)$ & $18 / 223(8.07 \%))$ \\
\hline$P$ value & $<0.0001$ & 0.0132 & 0.0022 \\
\hline Pregnant women & $120 / 504(23.81 \%)$ & $27 / 120(22.50 \%)$ & $8 / 27(29.62 \%)$ \\
\hline non-pregnant women & $142 / 528(26.90 \%)$ & $28 / 142(19.72 \%)$ & $2 / 28(7.14 \%)$ \\
\hline$P$ value & 0.2551 & 0.5817 & 0.0403 \\
\hline
\end{tabular}

RITS: respiratory tract infections; MPP: Mycoplasma pneumonia Pneumonia ; SMPP: severe Mycoplasma pneumoniae Pneumonia

Table 3 Clinical characteristics of MPP and SMPP in adults and children

\begin{tabular}{|llll|}
\hline & $\mathrm{MPP}(\mathrm{n}=198)$ & $\mathrm{SMPP}(\mathrm{n}=18)$ & $P$ value \\
\hline Clinical presentaion & & & \\
\hline Common symptoms(\%) & $198 / 198(100 \%)$ & $18 / 18(100 \%)$ & 1.0000 \\
\hline Extra-pulmonary symptoms(\%) & $20 / 198(10.15 \%)$ & $6 / 18(33.33 \%)$ & 0.0116 \\
\hline Laboratory detection & & & \\
\hline White blood cell (×10\%/L,range) & $6.3(2.8-16.4)$ & $10.9(3.9-20.1)$ & 0.0126 \\
\hline Neutrophil rate (\%, range) & $62.8(22.4-81.7)$ & $65.4(30.2-87.2)$ & 0.3801 \\
\hline Lymphocyte rate (\%,range) & $28.8(9.5-65.0)$ & $31.0(8.1-60.4)$ & 0.4661 \\
\hline C-reactive protein (mg/L,range) & $35.3(10.2-125.6)$ & $67.4(27.1-237.3)$ & $<0.0001$ \\
\hline LDH (U/L, range) & $309(170-620)$ & $447(150-1129)$ & 0.0032 \\
\hline Image manifestations & & & \\
\hline Unilobar infiltration(\%) & $117 / 198(59.09 \%)$ & $5 / 18(27.78 \%)$ & 0.0128 \\
\hline Multilobar infiltrates(\%) & $81 / 198(40.91 \%)$ & $13 / 18(72.22 \%)$ & 0.0128 \\
\hline Pleural effusion(\%) & $34 / 198(17.17 \%)$ & $8 / 18(33.33 \%)$ & 0.0103 \\
\hline
\end{tabular}

MPP:Mycoplasma pneumoniae pneumonia; SMMP: severe Mycoplasma pneumoniae pneumonia; Common symptoms: cough, sputum, headache, sore throat, fever, malaise, chest pain, trachypnea; extrapulmonary symptoms: skin rash, meningitis, mycocarditis, hemolytic anemia, myalgias digestive symptoms etc. 


\section{Figures}

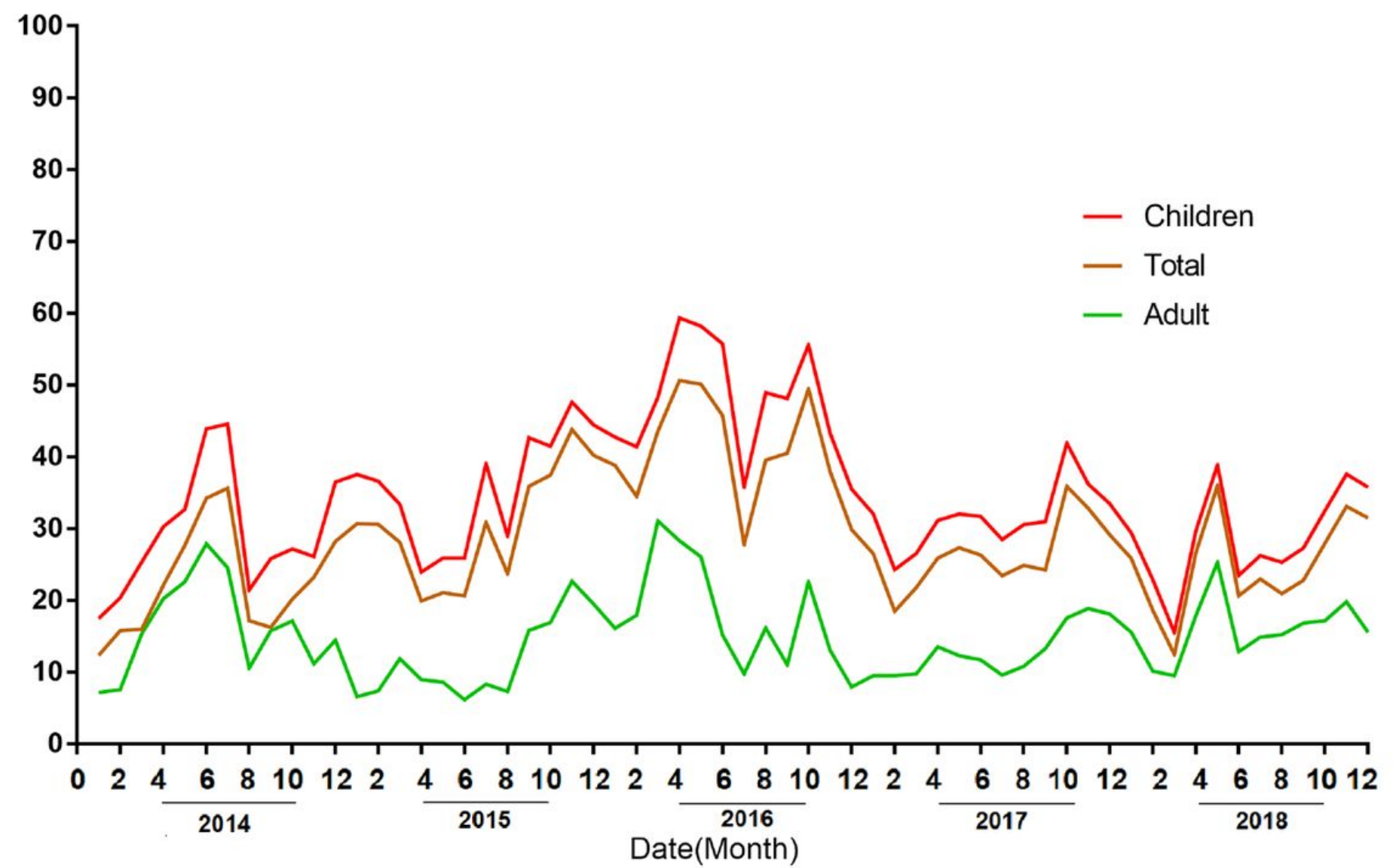

Figure 1

The prevalence trend of MP infection in adults and children from 2014 to 2018 . The MP RITs proportion was the highest in 2016 and lowest in 2018. The MP RITs proportion in children was significantly higher (approximately 2 folds) than that in adults in almost every month, and the positive rate trend of the two groups was consistent. 


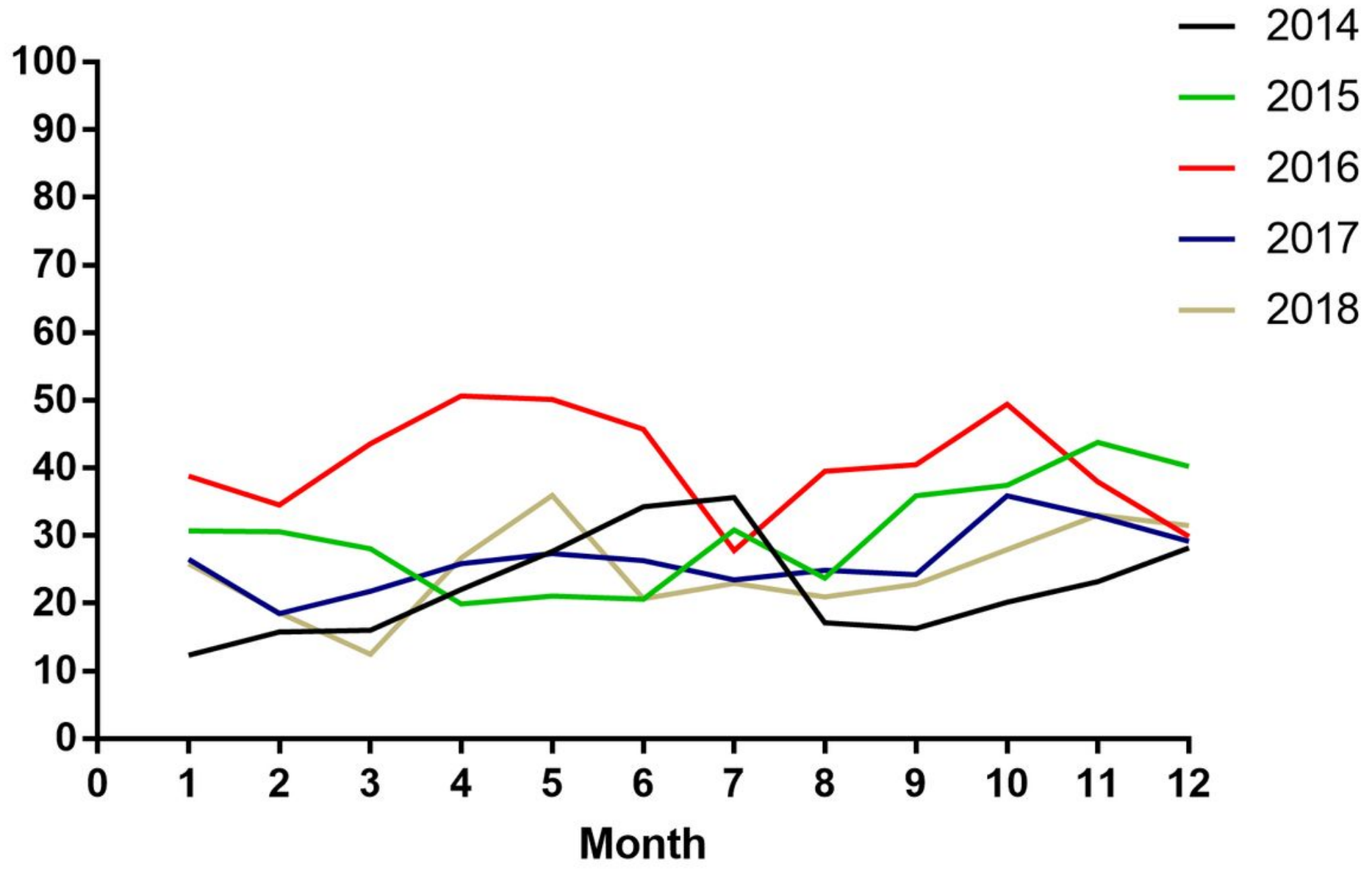

Figure 2

Season distributions of RITs with MP from 2014 to 2018. April to June and September to November were relatively peak months, especially in the epidemic year of 2016. 


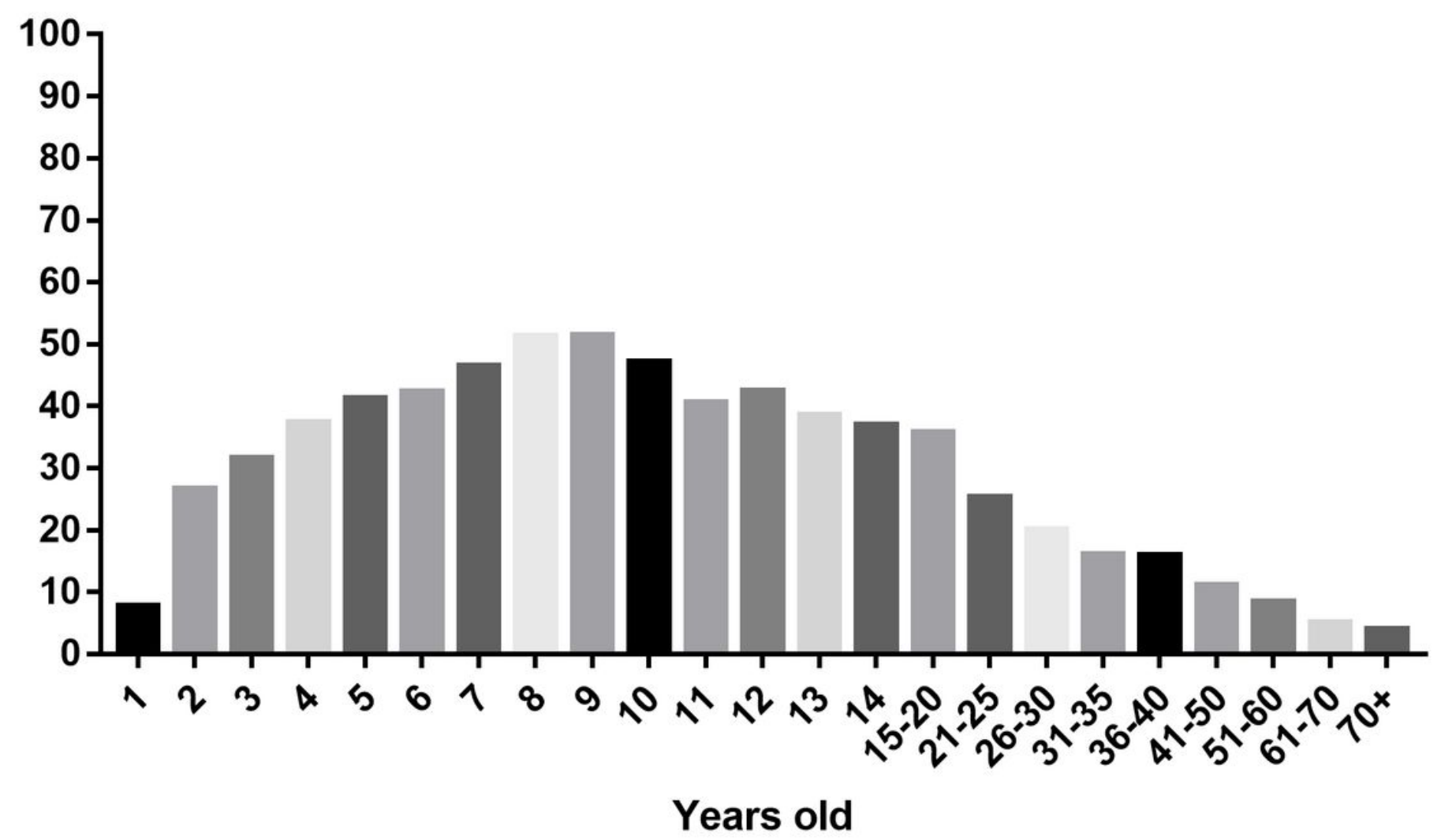

Figure 3

Age distributions of RITs with MP from 2014 to 2018. Children and adolescents were the main population of MP infection, of which aged 4-14 years old were the high-risk group. In adults, the MP RITs proportion decreased gradually after the age of 20 , and the MP RITs proportion of elderly patients was very low (less than $5 \%$ ). 
A
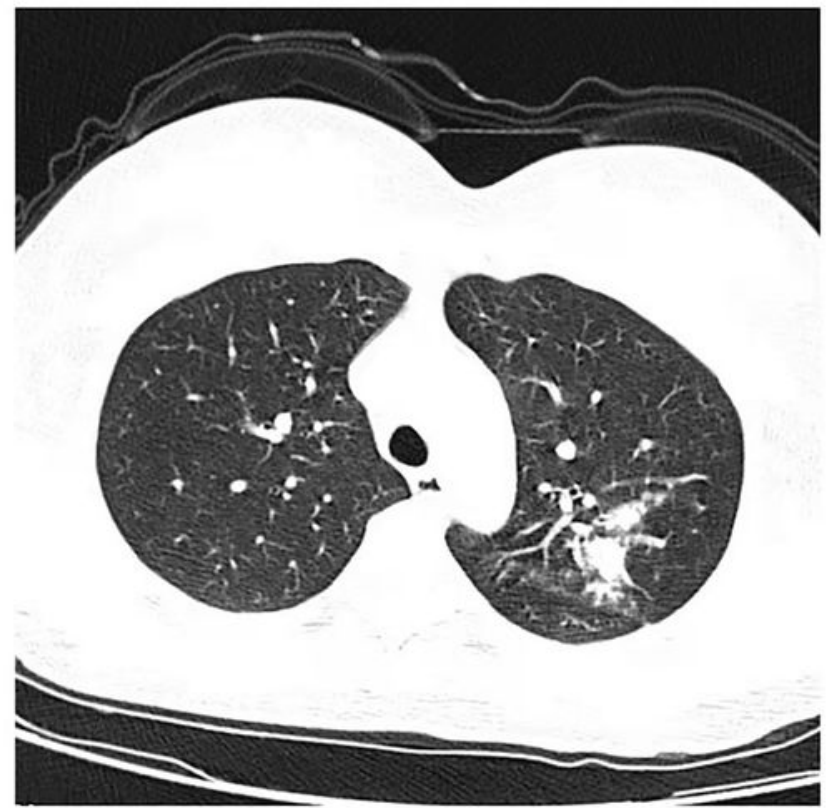

C

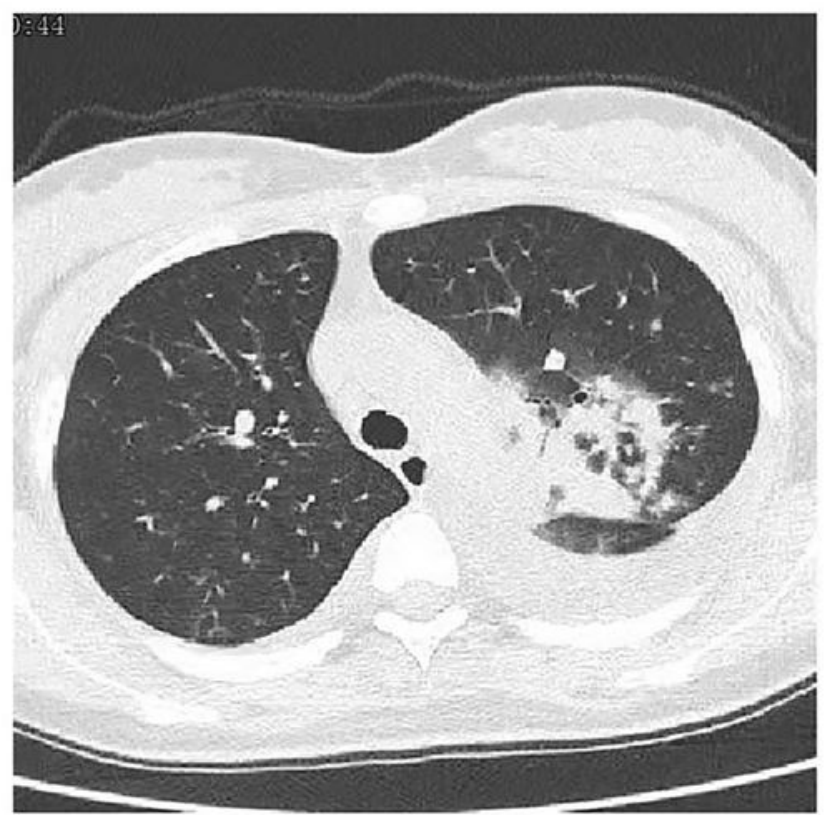

B
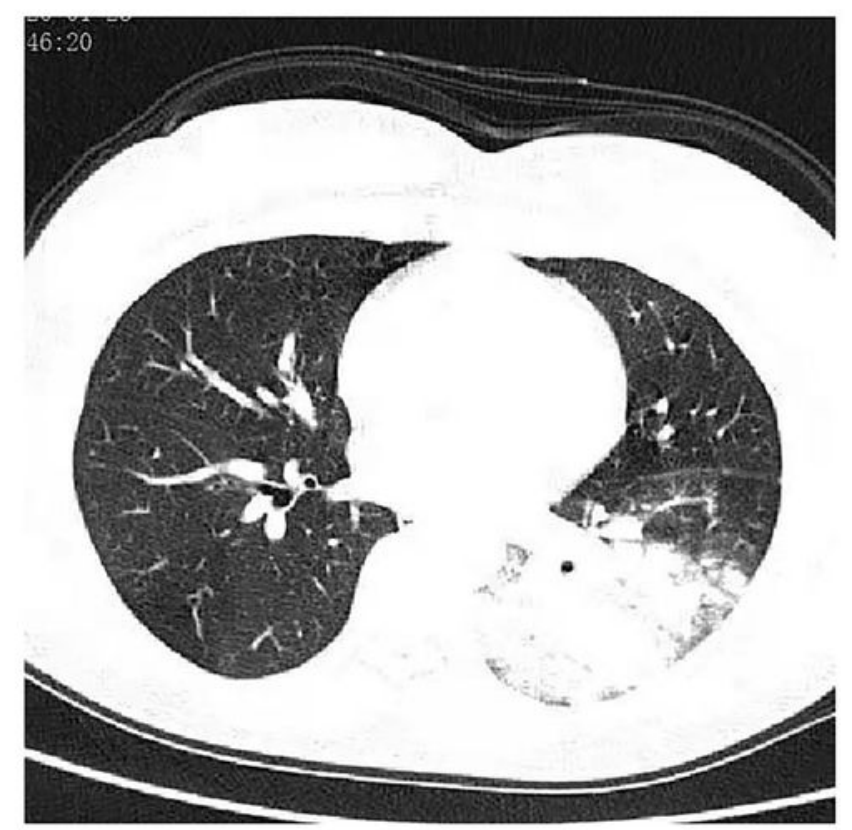

D

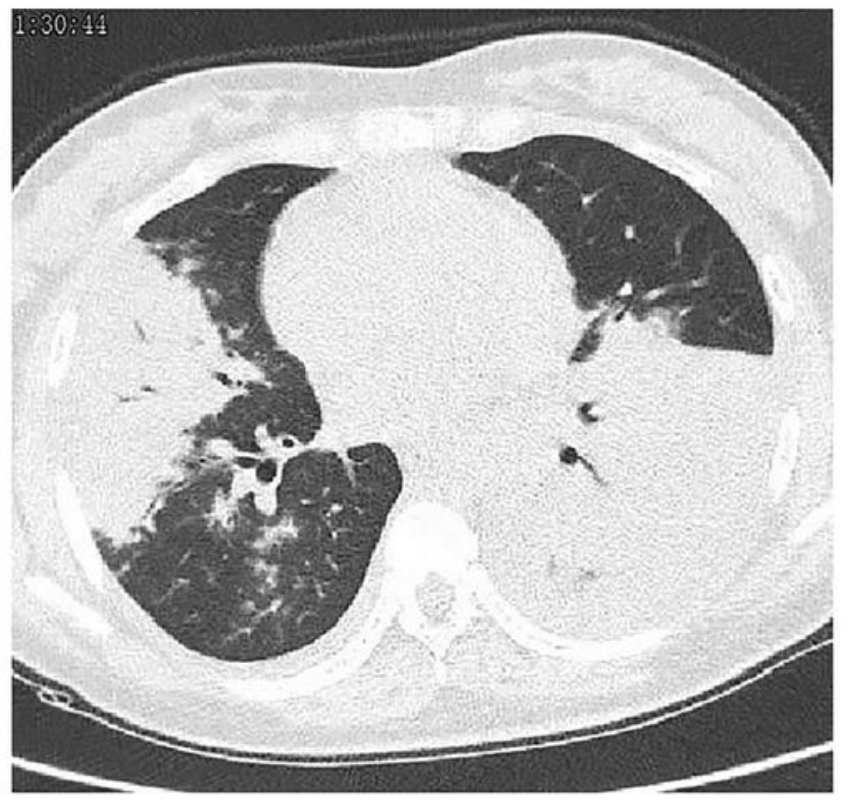

Figure 4

Representative CT images of SMMP in pregnant women A. B. Multilobar infiltration and pleural effusion were shown on different levels $\mathrm{C}$. One week later, the same level of imaging showed deteriorated mutilobar infiltration, atelectasis and increased pleural effusion. 